\title{
Three-valued simple games
}

\author{
M. Musegaas ${ }^{1}$ - P. E. M. Borm² - M. Quant ${ }^{2}$
}

(C) The Author(s) 2017. This article is an open access publication

\begin{abstract}
In this paper we study three-valued simple games as a natural extension of simple games. We analyze to which extent well-known results on the core and the Shapley value for simple games can be extended to this new setting. To describe the core of a three-valued simple game we introduce (primary and secondary) vital players, in analogy to veto players for simple games. Moreover, it is seen that the transfer property of Dubey (1975) can still be used to characterize the Shapley value for three-valued simple games. We illustrate three-valued simple games and the corresponding Shapley value in a parliamentary bicameral system.
\end{abstract}

Keywords Cooperative games · Three-valued simple games · Core · Vital players · Shapley value $\cdot$ Transfer property

\section{Introduction}

In this paper we analyze a class of transferable utility games, called three-valued simple games. The class of three-valued simple games is a natural extension of the class of simple games, introduced by Von Neumann and Morgenstern (1944) and widely applied in the literature to model decision rules in legislatures and other decisionmaking bodies. In a simple game, a coalition is either 'winning' or 'losing', i.e., there are two possible values for each coalition. The concept of three-valued simple games

$\triangle \quad$ M. Musegaas

musegaas@ese.eur.nl

1 Econometric Institute, Erasmus University Rotterdam, 1738, 3000 DR Rotterdam, The Netherlands

2 CentER and Department of Econometrics and Operations Research Tilburg University, 90153, 5000 LE Tilburg, The Netherlands 
goes one step further than simple games in the sense that there are three, instead of only two, possible values.

Simple games often represent simple voting games in which the way of determining which coalitions are winning is structured in the sense that each voter has two options: voting 'yes' or voting 'no'. Felsenthal and Machover (1997) generalized simple voting games by introducing ternary voting games, which recognizes abstention as an option next to the 'yes' and 'no' votes. Ternary voting games are extended by introducing $(j, k)$ simple voting games (see Freixas and Zwicker 2003) with several levels of approval in the input ( $j$ levels) and the output ( $k$ levels). Note that simple voting games correspond to the case $(j, k)=(2,2)$ and ternary voting games to $(j, k)=$ $(3,2)$. Freixas $(2005 b)$ generalized the Shapley-Shubik index (cf. Shapley and Shubik $1954)$ for $(j, k)$ simple voting games and provided a characterization for the class of $(j, 2)$ simple voting games. Other indices for (classes of) $(j, k)$ simple voting games are studied in Freixas (2005a) and Freixas (2012). Gibbard (1973) analyzed voting in a more general setting than $(j, k)$ simple voting but mainly focussed on the possible manipulation of voting schemes. Finally, we mention Hsiao and Raghavan (1993) who in the general framework of multi-choice cooperative games are the first to analyze input or effort levels on the part of the players in joining cooperating coalitions.

Note that there is a major conceptual difference between $(j, k)$ simple voting games and three-valued simple games. Although, of course, every three-valued simple game can be considered as a $(2,3)$ simple voting game, the definition of a three-valued simple game (and in fact of a simple game too) does not require a specification of the exact structure of how the values of the various coalitions are obtained. In $(j, k)$ simple voting games such a specification is provided in the definition. We will illustrate this conceptual difference in a more concrete example later on, namely Example 3.3.

This paper formally defines the class of three-valued simple games and focuses on analyzing the core and the Shapley value of these games. We study how the results for simple games can be extended to three-valued simple games. We extend the notion of veto players in simple games, to the notion of vital players, primary vital players and secondary vital pairs in three-valued simple games. It is known that in simple games the core is fully determined by the veto players. In a similar way, we introduce the vital core which fully depends on (primary and secondary) vital players. The vital core is shown to be a subset of the core. We discuss two classes of three-valued simple games such that the core and the vital core coincide.

Dubey (1975) characterized the Shapley value on the class of simple games. The essence of this characterization is the transfer property. We will show that the transfer property can also be used for a characterization of the Shapley value for three-valued simple games. In order to obtain this characterization we introduce a new axiom, called unanimity level efficiency. We prove that the combination of the axioms of efficiency, symmetry, the dummy property, the transfer property and unanimity level efficiency fully determines the Shapley value for a three-valued simple game. Moreover, also the logical independence of these five axioms is shown. At last, as an illustration, a parliamentary bicameral system is modelled as a three-valued simple game and analyzed on the basis of the Shapley value.

The organization of this paper is as follows. Section 2 formally introduces threevalued simple games. In Sect. 3 the core of three-valued simple games is investigated. 
Section 4 provides a characterization for the Shapley value on the class of three-valued simple games.

\section{Simple and three-valued simple games}

In this section we recall the formal definition of simple games and define three-valued simple games.

With $N$ a non-empty finite set of players, a transferable utility (TU) game is a function $v: 2^{N} \rightarrow \mathbb{R}$ which assigns a number to each coalition $S \in 2^{N}$, where $2^{N}$ denotes the collection of all subsets of $N$. By convention, $v(\emptyset)=0$. Let $\mathrm{TU}^{N}$ denote the class of all TU-games with player set $N$.

A game $v \in \mathrm{TU}^{N}$ is called simple if

(i) $v(S) \in\{0,1\}$ for all $S \subset N$,

(ii) $v(N)=1$,

(iii) $v(S) \leq v(T)$ for all $S, T \in 2^{N}$ with $S \subset T$ (monotonicity).

A coalition is winning if $v(S)=1$ and losing if $v(S)=0$. Let $\mathrm{SI}^{N}$ denote the class of all simple games with player set $N$.

In this paper we investigate a new subclass of TU-games, the class of three-valued simple games. A game $v \in \mathrm{TU}^{N}$ is called three-valued simple if

(i) $v(S) \in\{0,1,2\}$ for all $S \subset N$,

(ii) $v(N)=2$,

(iii) $v(S) \leq v(T)$ for all $S, T \in 2^{N}$ with $S \subset T$ (monotonicity).

Let $\mathrm{TSI}^{N}$ denote the class of all three-valued simple games with player set $N$. The concept of three-valued simple games goes one step further than simple games in the sense that there are three, instead of only two, possible values. Next to the value 0 , we have chosen the values 1 and 2 . Of course, the relative proportion between these two values may depend on the application at hand and the concept of three-valued simple games (and its results) can be generalized to three-valued TU-games with coalitional values 0,1 or $\beta$ with $\beta>1$.

\section{The core of three-valued simple games}

In this section we investigate the core of three-valued simple games. For this, we extend the concept of veto players in simple games, to the concept of vital players, primary vital players and secondary vital pairs in three-valued simple games.

The core $C(v)$ of a game $v \in \mathrm{TU}^{N}$ is defined as the set of all allocations $x \in \mathbb{R}^{N}$ such that $\sum_{i \in N} x_{i}=v(N)$ (efficiency) and $\sum_{i \in S} x_{i} \geq v(S)$ for all $S \subset N$ (stability). Hence, the core consists of all possible allocations of $v(N)$ for which no coalition has the incentive to leave the grand coalition. Consequently, if the core is empty, then it is not possible to find a stable allocation of $v(N)$.

Recall that for a simple game $v \in \mathrm{SI}^{N}$ the set of veto players are those players who belong to every coalition with value 1 , i.e.,

$$
\operatorname{veto}(v)=\bigcap\{S \mid v(S)=1\}
$$


In addition, the core of $v \in \mathrm{SI}^{N}$ is fully determined by its set of veto players, namely

$$
C(v)=\operatorname{Conv}\left(\left\{e^{\{i\}} \mid i \in \operatorname{veto}(v)\right\}\right),
$$

where for $S \in 2^{N} \backslash\{\emptyset\}$, the characteristic vector $e^{S} \in \mathbb{R}^{N}$ is defined as

$$
e_{i}^{S}= \begin{cases}1 & \text { if } i \in S \\ 0 & \text { otherwise }\end{cases}
$$

for all $i \in N$.

We characterize the core of a three-valued simple game using the concept of vital players which is similar to the concept of veto players in simple games. Proposition 3.1 below states that only the vital players of a three-valued simple game can receive a positive payoff in the core, while all other players receive zero. Here, for $v \in \operatorname{TSI}^{N}$ the set of vital players is defined by

$$
\operatorname{Vit}(v)=\bigcap\{S \mid v(S)=2\}
$$

Hence, the vital players are those players who belong to every coalition with value 2 .

Proposition 3.1 Let $v \in \mathrm{TSI}^{N}$. If $x \in C(v)$ and $i \in N \backslash \operatorname{Vit}(v)$, then $x_{i}=0$.

Proof Let $x \in C(v)$ and $i \in N \backslash \operatorname{Vit}(v)$. Since $i \notin \operatorname{Vit}(v)$, there exists a $T \subseteq N \backslash\{i\}$ with $v(T)=2$. Clearly, since $x \in C(v)$, we have $x \geq 0$. Then, because of efficiency and stability of $x \in C(v)$,

$$
x_{i} \leq v(N)-\sum_{j \in T} x_{j} \leq v(N)-v(T)=0,
$$

so $x_{i}=0$.

Using the concept of vital players, Proposition 3.2 provides a sufficient condition for emptiness of the core of a three-valued simple game.

Proposition 3.2 Let $v \in \operatorname{TSI}^{N}$. If $\operatorname{Vit}(v)=\emptyset$ or $v(N \backslash \operatorname{Vit}(v))>0$, then $C(v)=\emptyset .^{1}$

Proof First, assume $\operatorname{Vit}(v)=\emptyset$. Suppose $C(v) \neq \emptyset$ and let $x \in C(v)$. Then, from Proposition 3.1 we know $x_{i}=0$ for all $i \in N$. Consequently, $\sum_{i \in N} x_{i}=0$ which contradicts the efficiency condition of $x \in C(v)$.

\footnotetext{
1 Note that the condition is only a sufficient condition and not a necessary condition. Consider for example the game $v \in \operatorname{TSI}^{N}$, with $N=\{1,2,3\}$, given by

$$
v(S)= \begin{cases}2 & \text { if } S=N \\ 1 & \text { otherwise }\end{cases}
$$
}

Then, $C(v)=\emptyset$ but $\operatorname{Vit}(v)=N \neq \emptyset$ and $v(N \backslash \operatorname{Vit}(v))=v(\emptyset)=0$. 
Second, assume $v(N \backslash \operatorname{Vit}(v))>0$. Suppose $C(v) \neq \emptyset$ and let $x \in C(v)$. Then, from Proposition 3.1 we know $x_{i}=0$ for all $i \in N \backslash \operatorname{Vit}(v)$ and therefore $\sum_{i \in N \backslash \operatorname{Vit}(v)} x_{i}=$ $0<v(N \backslash \operatorname{Vit}(v))$, which contradicts the stability condition of $x \in C(v)$.

From Proposition 3.2 it follows that only the set of permissible three-valued simple games may have a non-empty core, where a game $v \in \operatorname{TSI}^{N}$ is called permissible if the following two conditions are satisfied:

(i) $\operatorname{Vit}(v) \neq \emptyset$,

(ii) $v(N \backslash \operatorname{Vit}(v))=0$.

As a consequence, from now on we focus only on the set of permissible three-valued simple games and define for every permissible three-valued simple game, a reduced game where the player set is reduced to the set of vital players. We define this reduced game in such a way that the core of a permissible three-valued simple game equals the core of the reduced game, when extended with zeros for all players outside the set of vital players (see Proposition 3.4).

For a permissible game $v \in \operatorname{TSI}^{N}$ the reduced three-valued simple game $v_{r} \in$ $\mathrm{TU}^{\mathrm{Vit}(v)}$ is defined by

$$
v_{r}(S)=v(S \cup(N \backslash \operatorname{Vit}(v))),
$$

for all $S \subseteq \operatorname{Vit}(v)$. The following proposition states that a reduced permissible game $v_{r}$ is also a three-valued simple game and, interestingly, allows for only one coalition with value 2 .

Proposition 3.3 Let $v \in \mathrm{TSI}^{N}$ be permissible. Then, $v_{r} \in \mathrm{TSI}^{\mathrm{Vit}(v)}$ with

$$
v_{r}(S) \in\{0,1\}
$$

for all $S \subset \operatorname{Vit}(v)$.

Proof From the definition of $v_{r}$ it immediately follows that $v_{r} \in \mathrm{TSI}^{\mathrm{Vit}(v)}$. Suppose that there exists an $S \subset \operatorname{Vit}(v)$ with $v_{r}(S)=2$. Then $v(S \cup(N \backslash \operatorname{Vit}(v)))=2$ and consequently, using the definition of $\operatorname{Vit}(v)$, we have $\operatorname{Vit}(v) \subseteq S$, which is a contradiction.

In a reduced three-valued simple game the number of coalitions with value 2 is reduced to one, only the grand coalition has value 2, and thus $\operatorname{Vit}\left(v_{r}\right)=\operatorname{Vit}(v)$. This property makes it easier to characterize the core of reduced three-valued simple games compared to non-reduced three-valued simple games.

For a permissible game $v \in \mathrm{TSI}^{N}$ and for an $x \in \mathbb{R}^{\operatorname{Vit}(v)}$ we define $\bar{x}^{0} \in \mathbb{R}^{N}$ as

$$
\bar{x}_{i}^{0}= \begin{cases}x_{i} & \text { if } i \in \operatorname{Vit}(v), \\ 0 & \text { if } i \in N \backslash \operatorname{Vit}(v) .\end{cases}
$$

For a set $X \subseteq \mathbb{R}^{\operatorname{Vit}(v)}$, we define $\bar{X}^{0} \subseteq \mathbb{R}^{N}$ as $\bar{X}^{0}=\left\{\bar{x}^{0} \mid x \in X\right\}$ 
Proposition 3.4 Let $v \in T S I^{N}$ be permissible. Then,

$$
C(v)={\overline{C\left(v_{r}\right)}}^{0}
$$

Proof (" $\subseteq$ ") Let $x \in C(v)$ and let $S \subseteq \operatorname{Vit}(v)$. From Proposition 3.1 we have

$$
\sum_{i \in S} x_{i}=\sum_{i \in S \cup(N \backslash \operatorname{Vit}(v))} x_{i} \geq v(S \cup(N \backslash \operatorname{Vit}(v)))=v_{r}(S),
$$

where the inequality follows from stability of $x \in C(v)$. Because of efficiency of $x \in C(v)$ and due to Proposition 3.1 we have

$$
\sum_{i \in \operatorname{Vit}(v)} x_{i}=\sum_{i \in N} x_{i}=v(N)=2=v_{r}(\operatorname{Vit}(v))
$$

where the last equality follows from Proposition 3.3. Hence, $x \in{\overline{C\left(v_{r}\right)}}^{0}$.

(" $\supseteq$ ") Let $x \in \overline{C\left(v_{r}\right)}$ and let $S \subseteq N$. Then,

$$
\sum_{i \in S} x_{i}=\sum_{i \in S \cap \operatorname{Vit}(v)} x_{i} \geq v_{r}(S \cap \operatorname{Vit}(v))=v((S \cap \operatorname{Vit}(v)) \cup(N \backslash \operatorname{Vit}(v))) \geq v(S),
$$

where the first inequality follows from stability of $x \in C\left(v_{r}\right)$ and the second inequality follows from monotonicity of $v$ and the fact that $S \subseteq(S \cap \operatorname{Vit}(v)) \cup(N \backslash \operatorname{Vit}(v))$. Because of efficiency of $x \in C\left(v_{r}\right)$ we have

$$
\sum_{i \in N} x_{i}=\sum_{i \in \operatorname{Vit}(v)} x_{i}=v_{r}(\operatorname{Vit}(v))=2=v(N)
$$

where the penultimate equality follows from Proposition 3.3. Hence, $x \in C(v)$.

Proposition 3.4 states that the core of a permissible three-valued simple game follows from the core of the corresponding reduced game by extending the vectors with zeros for the non-vital players. This proposition is illustrated in the following example.

Example 3.1 Let $N=\{1,2,3,4\}$ and consider the game $v \in \mathrm{TSI}^{N}$ given by

$$
v(S)= \begin{cases}2 & \text { if } S \in\{\{1,2,3\}, N\} \\ 1 & \text { if } S \in\{\{1,2\},\{2,3\},\{1,2,4\},\{2,3,4\}\} \\ 0 & \text { otherwise. }\end{cases}
$$

Note that $v$ is permissible since $\operatorname{Vit}(v)=\{1,2,3\} \neq \varnothing$ and $v(N \backslash \operatorname{Vit}(v))=v(\{4\})=$ 0 . The corresponding reduced three-valued simple game $v_{r}$ is given in Table 1 . 
Table 1 Reduced game $v_{r}$ of the game $v$ in Example 3.1

\begin{tabular}{llllllll}
\hline$S$ & $\{1\}$ & $\{2\}$ & $\{3\}$ & $\{1,2\}$ & $\{1,3\}$ & $\{2,3\}$ & $\{1,2,3\}$ \\
\hline$v_{r}(S)$ & 0 & 0 & 0 & 1 & 0 & 1 & 2 \\
\hline
\end{tabular}

Since

$$
C\left(v_{r}\right)=\operatorname{Conv}\left(\left(\begin{array}{l}
0 \\
2 \\
0
\end{array}\right),\left(\begin{array}{l}
1 \\
1 \\
0
\end{array}\right),\left(\begin{array}{l}
0 \\
1 \\
1
\end{array}\right),\left(\begin{array}{l}
1 \\
0 \\
1
\end{array}\right)\right)
$$

we have, according to Proposition 3.4, that

$$
C(v)=\operatorname{Conv}\left(\left(\begin{array}{l}
0 \\
2 \\
0 \\
0
\end{array}\right),\left(\begin{array}{l}
1 \\
1 \\
0 \\
0
\end{array}\right),\left(\begin{array}{l}
0 \\
1 \\
1 \\
0
\end{array}\right),\left(\begin{array}{l}
1 \\
0 \\
1 \\
0
\end{array}\right)\right)
$$

As Example 3.1 suggests, the extreme points of the core of three-valued simple games have a specific structure which we will describe using the notion of the vital core. The extreme points depend in particular on the set of vital players that belong to every coalition with value 1 or 2 in $v_{r}$ and the set of pairs of vital players such that for every coalition with value 1 in $v_{r}$ at least one player of such a pair belongs to the coalition. For a permissible three-valued simple game $v \in \mathrm{TSI}^{N}$ we define the set of primary vital players of $v$ by

$$
\operatorname{PVit}(v)=\bigcap\left\{S \subseteq \operatorname{Vit}(v) \mid v_{r}(S) \in\{1,2\}\right\}
$$

and define the set of secondary vital pairs of $v$ by

$\operatorname{SVit}(v)=\left\{\{i, j\} \subseteq \operatorname{Vit}(v) \backslash \operatorname{PVit}(v) \mid i \neq j,\{i, j\} \cap S \neq \emptyset\right.$ for all $S$ with $\left.v_{r}(S)=1\right\}$

Using the primary vital players and the secondary vital pairs, the vital core $V C(v)$ of a permissible game $v \in \mathrm{TSI}^{N}$ is defined by

$$
\begin{aligned}
V C(v)= & \operatorname{Conv}\left(\left\{2 e^{\{i\}} \mid i \in \operatorname{PVit}(v)\right\}\right. \\
& \cup\left\{e^{\{i, j\}} \mid i \in \operatorname{PVit}(v), j \in \operatorname{Vit}(v) \backslash \operatorname{PVit}(v)\right\} \\
& \left.\cup\left\{e^{\{i, j\}} \mid\{i, j\} \in \operatorname{SVit}(v)\right\}\right) .
\end{aligned}
$$

The vital core ${ }^{2}$ is a subset of the core as is seen in the following theorem.

\footnotetext{
2 Note that the concept of the vital core can easily be generalized for three-valued TU-games with coalitional values 0,1 or $\beta$ with $\beta>1$.
} 
Theorem 3.5 Let $v \in T S I^{N}$ be permissible. Then, ${ }^{3}$

$$
V C(v) \subseteq C(v)
$$

Proof Due to Proposition 3.4 together with the fact that $C(v)$ is a convex set, it is sufficient to show that $2 e^{\{i\}} \in C\left(v_{r}\right)$ for all $i \in \operatorname{PVit}(v), e^{\{i, j\}} \in C\left(v_{r}\right)$ for all $i \in \operatorname{PVit}(v)$ and $j \in \operatorname{Vit}(v) \backslash \operatorname{PVit}(v)$, and $e^{\{i, j\}} \in C\left(v_{r}\right)$ for all $\{i, j\} \in \operatorname{SVit}(v){ }^{4}$

Let $i \in \operatorname{PVit}(v)$ and $S \subset \operatorname{Vit}(v)$. If $i \in S$, then

$$
\sum_{k \in S} 2 e_{k}^{\{i\}}=2>1 \geq v_{r}(S) .
$$

If $i \notin S$, then $v_{r}(S)=0$ and

$$
\sum_{k \in S} 2 e_{k}^{\{i\}}=0=v_{r}(S) .
$$

Moreover, $\sum_{k \in \operatorname{Vit}(v)} 2 e_{k}^{\{i\}}=2=v_{r}(\operatorname{Vit}(v))$. Hence, $2 e^{\{i\}}$ belongs to $C\left(v_{r}\right)$.

Next, let $i \in \operatorname{PVit}(v), j \in \operatorname{Vit}(v) \backslash \operatorname{PVit}(v)$ and $S \subset \operatorname{Vit}(v)$. If $i \in S$, then

$$
\sum_{k \in S} e_{k}^{\{i, j\}} \geq 1 \geq v_{r}(S) .
$$

If $i \notin S$, then $v_{r}(S)=0$ and

$$
\sum_{k \in S} e_{k}^{\{i, j\}} \geq 0=v_{r}(S)
$$

Moreover, $\sum_{k \in \operatorname{Vit}(v)} e_{k}^{\{i, j\}}=2=v_{r}(\operatorname{Vit}(v))$. Hence, $e^{\{i, j\}}$ belongs to $C\left(v_{r}\right)$.

Finally, let $\{i, j\} \in \operatorname{SVit}(v)$ and let $S \subset \operatorname{Vit}(v)$. If $S \cap\{i, j\} \neq \varnothing$, then

$$
\sum_{k \in S} e_{k}^{\{i, j\}} \geq 1 \geq v_{r}(S)
$$

If $S \cap\{i, j\}=\emptyset$, then $v_{r}(S)=0$ and

$$
\sum_{k \in S} e_{k}^{\{i, j\}}=0=v_{r}(S) .
$$

Moreover, $\sum_{k \in \operatorname{Vit}(v)} e_{k}^{\{i, j\}}=2=v_{r}(\operatorname{Vit}(v))$. Hence, $e^{\{i, j\}}$ belongs to $C\left(v_{r}\right)$.

\footnotetext{
3 This theorem can be generalized for three-valued TU-games with coalitional values 0,1 or $\beta$ with $\beta \geq 2$.

${ }^{4}$ By the nature of the restricted game $v_{r}$ we should actually write $2 e^{\{i\}} \mid \operatorname{Vit}(v)$ and $e^{\{i, j\}} \mid \operatorname{Vit}(v)$ since we consider the restriction of the vectors $2 e^{\{i\}}$ and $e^{\{i, j\}}$ to $\operatorname{Vit}(v)$.
} 
As the following example shows, the vital core and the core coincide for some three-valued simple games.

Example 3.2 Reconsider the three-valued simple game of Example 3.1. From the reduced game $v_{r}$ (see Table 1) it follows that

$$
\operatorname{PVit}(v)=\{2\}
$$

and

$$
\operatorname{SVit}(v)=\{\{1,3\}\}
$$

Therefore, the vital core of $v$ is given by

$$
V C(v)=\operatorname{Conv}\left(\left(\begin{array}{l}
0 \\
2 \\
0 \\
0
\end{array}\right),\left(\begin{array}{l}
1 \\
1 \\
0 \\
0
\end{array}\right),\left(\begin{array}{l}
0 \\
1 \\
1 \\
0
\end{array}\right),\left(\begin{array}{l}
1 \\
0 \\
1 \\
0
\end{array}\right)\right)
$$

and, using the results from Example 3.1, we have $V C(v)=C(v)$.

The result of the previous example is generalized in Theorem 3.6 below which shows that the core and the vital core coincide for the class of convex three-valued simple games. Here a game $v \in \mathrm{TU}^{N}$ is called convex if

$$
v(S \cup\{i\})-v(S) \leq v(T \cup\{i\})-v(T),
$$

for all $S, T \in 2^{N}, i \in N$ such that $S \subset T \subseteq N \backslash\{i\}$. From Shapley (1971) and Ichiishi (1981) it follows that $v \in \mathrm{TU}^{N}$ is convex if and only if

$$
C(v)=\operatorname{Conv}\left(\left\{m^{\sigma}(v) \mid \sigma \in \Pi(N)\right\}\right),
$$

where $\Pi(N)=\{\sigma: N \rightarrow\{1, \ldots,|N|\} \mid \sigma$ is bijective $\}$ is the set of all orders on $N$ and the marginal vector $m^{\sigma}(v) \in \mathbb{R}^{N}$, for $\sigma \in \Pi(N)$, is defined by

$$
m_{i}^{\sigma}(v)=v(\{j \in N \mid \sigma(j) \leq \sigma(i)\})-v(\{j \in N \mid \sigma(j)<\sigma(i)\}),
$$

for all $i \in N$.

Theorem 3.6 Let $v \in \operatorname{TSI}^{N}$ be convex. ${ }^{5}$ Then,

$$
C(v)=V C(v) .
$$

\footnotetext{
5 Note that every convex three-valued simple game is permissible because every convex game has a nonempty core.
} 
Proof From Theorem 3.5 we already know $V C(v) \subseteq C(v)$, so we only need to prove $C(v) \subseteq V C(v)$. Together with Proposition 3.4 and the fact $V C(v)={\overline{V C\left(v_{r}\right)}}^{0}$, it suffices to prove

$$
C\left(v_{r}\right) \subseteq V C\left(v_{r}\right)
$$

Note that because $v$ is convex and due to the definition of a reduced game, also $v_{r}$ is convex. Therefore, because of (1) and because the vital core is a convex set, it suffices to show that

$$
\begin{aligned}
m^{\sigma}\left(v_{r}\right) & \in\left\{2 e^{\{i\}} \mid i \in \operatorname{PVit}\left(v_{r}\right)\right\} \cup\left\{e^{\{i, j\}} \mid i \in \operatorname{PVit}\left(v_{r}\right), j \in \operatorname{Vit}\left(v_{r}\right) \backslash \operatorname{PVit}\left(v_{r}\right)\right\} \\
& \cup\left\{e^{\{i, j\}} \mid\{i, j\} \in \operatorname{SVit}\left(v_{r}\right)\right\},
\end{aligned}
$$

for all $\sigma \in \Pi(\operatorname{Vit}(v)){ }^{6}$

Let $\sigma \in \Pi(\operatorname{Vit}(v))$. Since $v_{r}(S) \in\{0,1\}$ for all $S \subset \operatorname{Vit}(v), v_{r}(\operatorname{Vit}(v))=2$ and $v_{r}$ is monotonic, $m^{\sigma}\left(v_{r}\right)$ either contains one two with all other coordinates zero (Case 1) or it contains two ones with all other coordinates zero (Case 2). We thus distinguish between these two cases.

Case 1: $\left[m^{\sigma}\left(v_{r}\right)=2 e^{\{i\}}\right.$ for some $\left.i \in \operatorname{Vit}(v)\right]$

Set

$$
P=\{k \in \operatorname{Vit}(v) \mid \sigma(k)<\sigma(i)\} .
$$

Then, $v_{r}(P)=0$ and $v_{r}(P \cup\{i\})=2$. Since in a reduced three-valued simple game there is only one coalition, namely the grand coalition $\operatorname{Vit}(v)$, with value 2 , we have $P \cup\{i\}=\operatorname{Vit}(v)$ and thus $P=\operatorname{Vit}(v) \backslash\{i\}$. Therefore, from monotonicity it follows that $v_{r}(S)=0$ for all $S \subseteq \operatorname{Vit}(v) \backslash\{i\}$. Hence, $i \in S$ for every $S \subseteq \operatorname{Vit}(v)$ with $v_{r}(S) \in\{1,2\}$ and thus $i \in \operatorname{PVit}\left(v_{r}\right)$. Consequently, we have $m^{\sigma}\left(v_{r}\right)=2 e^{\{i\}}$ with $i \in \operatorname{PVit}\left(v_{r}\right)$.

Case 2: $\left[m^{\sigma}\left(v_{r}\right)=e^{\{i, j\}}\right.$ for some $\left.i, j \in \operatorname{Vit}(v), i \neq j\right]$

Without loss of generality assume that $\sigma(i)<\sigma(j)$. Set

$$
P=\{k \in \operatorname{Vit}(v) \mid \sigma(k)<\sigma(i)\} .
$$

Then, $v_{r}(P)=0$ and $v_{r}(P \cup\{i\})=1$. Since $v_{r}(P \cup\{i\})=1$ and $j \notin P \cup\{i\}$, we have $j \notin \operatorname{PVit}\left(v_{r}\right)$. We distinguish from now on between another two cases: $i \in \operatorname{PVit}\left(v_{r}\right)$ (Case 2(i)) and $i \notin \operatorname{PVit}\left(v_{r}\right)$ (Case 2(ii)).

Case 2(i): $\left[i \in \operatorname{PVit}\left(v_{r}\right)\right]$

\footnotetext{
6 Note that $\operatorname{Vit}(v)$ is the player set in $v_{r}$. Therefore, in the proof of Theorem 3.6 we write Vit $(v)$ if we talk about the player set of the game $v_{r}$ and we write $\operatorname{Vit}\left(v_{r}\right)$ if we talk about the vital players in the game $v_{r}$. However, actually $\operatorname{Vit}(v)=\operatorname{Vit}\left(v_{r}\right)$. Moreover, note that also $\left(v_{r}\right)_{r}=v_{r}$.
} 
Then, we have $m^{\sigma}\left(v_{r}\right)=e^{\{i, j\}}$ with $i \in \operatorname{PVit}\left(v_{r}\right)$ and $j \in \operatorname{Vit}\left(v_{r}\right) \backslash \operatorname{PVit}\left(v_{r}\right)$.

Case 2(ii): $\left[i \notin \operatorname{PVit}\left(v_{r}\right)\right]$

Then, we need to show $\{i, j\} \in \operatorname{SVit}\left(v_{r}\right)$. Since $\{i, j\} \subseteq \operatorname{Vit}\left(v_{r}\right) \backslash \operatorname{PVit}\left(v_{r}\right)$, it remains to show $\{i, j\} \cap S \neq \emptyset$ for all $S \subset \operatorname{Vit}(v)$ with $v_{r}(S)=1$. Suppose for the sake of contradiction that there exists a coalition $S \subset \operatorname{Vit}(v)$ with $v_{r}(S)=1$ and $\{i, j\} \cap S=\emptyset$. Then, due to monotonicity and the fact that $S \cup P \cup\{i\} \neq \operatorname{Vit}(v)$ because $j \notin S$ and $j \notin P$, we have $v_{r}(S \cup P)=1$ and $v_{r}(S \cup P \cup\{i\})=1$. As a consequence,

$$
v_{r}(S \cup P \cup\{i\})-v_{r}(S \cup P)=0,
$$

while

$$
v_{r}(P \cup\{i\})-v_{r}(P)=1,
$$

which is a contradiction with $v_{r}$ being convex. Consequently, $m^{\sigma}\left(v_{r}\right)=e^{\{i, j\}}$ with $\{i, j\} \in \operatorname{SVit}\left(v_{r}\right)$.

An example of a class of convex three-valued simple games is the class of double unanimity games. Therefore, Theorem 3.6 implies that the core and the vital core coincide for the class of double unanimity games. For $T_{1}, T_{2} \in 2^{N} \backslash\{\emptyset\}$, we define the double unanimity game $u_{T_{1}, T_{2}} \in \mathrm{TSI}^{N}$ by

$$
u_{T_{1}, T_{2}}(S)= \begin{cases}2 & \text { if } T_{1} \subseteq S \quad \text { and } \quad T_{2} \subseteq S, \\ 1 & \text { if } T_{1} \subseteq S, T_{2} \not \subseteq S \text { or } T_{1} \nsubseteq \subseteq S, T_{2} \subseteq S, \\ 0 & \text { otherwise }\end{cases}
$$

for all $S \in 2^{N}$. Note that a three-value simple double unanimity game is a natural extension of a simple unanimity game $u_{T} \in \mathrm{SI}^{N}$, with $T \in 2^{N} \backslash\{\emptyset\}$, defined by

$$
u_{T}(S)= \begin{cases}1 & \text { if } T \subseteq S, \\ 0 & \text { otherwise }\end{cases}
$$

for all $S \in 2^{N}$.

The following example illustrates an interactive cooperative operations research situation based on a minimum coloring problem [as introduced by Deng et al. (1999)] that can be modelled as a three-valued simple game. Theorem 3.7 (cf. Musegaas et al. 2016) shows that the core and the vital core coincide for a subclass of minimum coloring games. Note that minimum coloring games are not necessarily convex and thus Theorem 3.7 is not a direct consequence of Theorem 3.6.

Example 3.3 Consider a set of agents $\{1,2,3,4\}$ who all need access to some facility. However, for some reason, some agents cannot have access to the same facility. The pairs of agents that are in conflict in this way are assumed to be $\{1,3\},\{1,4\}$ and $\{2,4\}$. This situation can be modelled by the conflict graph in Fig. 1. The total costs are assumed to be linearly increasing in the number of facilities used, so the aim is to 
Fig. 1 The conflict graph of Example 3.3

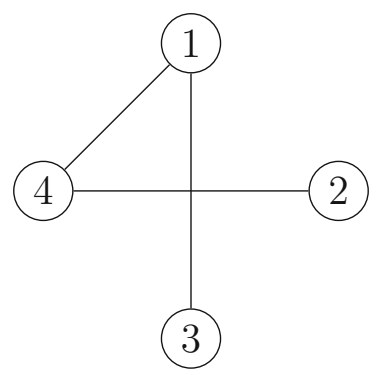

find the minimum number of facilities that can serve all agents. Clearly, the optimal facility allocation uses two facilities: players 1 and 2 have access to one facility, say facility $A$, and players 3 and 4 have access to another facility, say facility $B$.

If we assume that in the initial situation no agents share facilities (and thus four facilities are used), then cooperation in sharing facilities between non-conflicting agents will lead to cost savings. To analyze how to divide the minimal joint costs among the agents a minimum coloring game can be defined. In this minimum coloring game the value of a coalition is equal to the maximal number of facilities a coalition can save by means of sharing facilities. For instance, in our example, the value of the grand coalition will be 2 , because initially four facilities were used and by means of optimal cooperation only two facilities are needed. It turns out that the corresponding minimum coloring game $v \in \mathrm{TU}^{N}$ is a three-valued simple game and is given by

$$
v(S)= \begin{cases}2 & \text { if } S=N, \\ 1 & \text { if }\{1,2\} \subseteq S \quad \text { or }\{2,3\} \subseteq S \quad \text { or }\{3,4\} \subseteq S, \quad \text { and } \quad S \neq N \\ 0 & \text { otherwise, }\end{cases}
$$

for all $S \subseteq N$.

Note that it is assumed that all players would want to cooperate and thus in the corresponding three-valued simple game it is analyzed how to divide the maximal joint cost savings due to cooperation among the agents. In order to find a fair allocation of these cost savings, the values of the subcoalitions are taken into account. This illustrates the conceptual difference between three-valued simple games and voting games, where the focus is more on how decision are made instead of taking into account the worth/power of subcoalitions.

Theorem 3.7 (cf. Musegaas et al. 2016). Let $v \in T S I^{N}$ be a minimum coloring game induced by a perfect graph. ${ }^{7}$ Then,

$$
C(v)=V C(v) .
$$

\footnotetext{
7 Without going into details, a perfect graph is a graph in which the chromatic number of every induced subgraph equals the clique number of that subgraph.
} 
Example 3.4 illustrates that there exist three-valued simple games for which the vital core is a strict non-empty subset of the core.

Example 3.4 Let $v \in \operatorname{TSI}^{N}$ be given by $N=\{1, \ldots, 7\}$ and

$v(S)=\left\{\begin{aligned} 2 \quad & \text { if } S \in\{N \backslash\{6\}, N \backslash\{7\}, N\}, \\ 1 \quad & \text { if } S \notin\{N \backslash\{6\}, N \backslash\{7\}, N\} \text { and }\{1,3,5\} \subseteq S \text { or }\{3,4,5\} \subseteq S \text { or } \\ & \{1,2,3,6\} \subseteq S \text { or }\{1,3,4,7\} \subseteq S \text { or }\{2,3,4,6,7\} \subseteq S, \\ 0 & \text { otherwise. }\end{aligned}\right.$

Note that $v$ is permissible since $\operatorname{Vit}(v)=\{1,2,3,4,5\} \neq \varnothing$ and $v(N \backslash \operatorname{Vit}(v))=$ $v(\{6,7\})=0$. Hence, $v_{r} \in \operatorname{TSI}^{\operatorname{Vit}(v)}$ is given by

$$
v_{r}(S)= \begin{cases}2 \quad & \text { if } S=\operatorname{Vit}(v), \\ 1 \quad & \text { if } S \in\{\{1,2,3\},\{1,3,4\},\{1,3,5\},\{2,3,4\},\{3,4,5\} \\ & \{1,2,3,4\},\{1,2,3,5\},\{1,3,4,5\},\{2,3,4,5\}\} \\ 0 \quad \text { otherwise. }\end{cases}
$$

From this it follows that

$$
\operatorname{PVit}(v)=\{3\}
$$

and

$$
\operatorname{SVit}(v)=\{\{1,4\}\}
$$

Consequently,

$$
\left.V C(v)=\operatorname{Conv}\left(\begin{array}{l}
0 \\
0 \\
2 \\
0 \\
0 \\
0 \\
0
\end{array}\right),\left(\begin{array}{l}
1 \\
0 \\
1 \\
0 \\
0 \\
0 \\
0
\end{array}\right),\left(\begin{array}{l}
0 \\
1 \\
1 \\
0 \\
0 \\
0 \\
0
\end{array}\right),\left(\begin{array}{l}
0 \\
0 \\
1 \\
1 \\
0 \\
0 \\
0
\end{array}\right),\left(\begin{array}{l}
0 \\
0 \\
1 \\
0 \\
1 \\
0 \\
0
\end{array}\right),\left(\begin{array}{l}
1 \\
0 \\
0 \\
1 \\
0 \\
0 \\
0
\end{array}\right)\right) .
$$

Note that $\left(0, \frac{1}{2}, \frac{1}{2}, \frac{1}{2}, \frac{1}{2}, 0,0\right)$ belongs to $C(v)$, but does not belong to $V C(v)$ and thus $V C(v) \subset C(v)$.

\section{The Shapley value for three-valued simple games}

In this section we analyze the Shapley value for three-valued simple games. In the context of simple games and three-valued simple games, a one-point solution concept 
like the Shapley value can be interpreted as a measure for the relative influence of each player.

A one-point solution concept $f$ on the class $\mathrm{G}^{N}$ with $\mathrm{G}^{N} \subseteq \mathrm{TU}^{N}$ is a function $f: \mathrm{G}^{N} \rightarrow \mathbb{R}^{N}$. The Shapley value (Shapley 1953) is a solution concept on $\mathrm{TU}^{N}$ defined by

$$
\Phi_{i}(v)=\frac{1}{|N| !} \sum_{\sigma \in \Pi(N)} m^{\sigma}(v),
$$

for all $v \in \mathrm{TU}^{N}$, where a marginal vector $m^{\sigma}(v)$ with $\sigma \in \Pi(N)$ is as defined in (2). A characterization of the Shapley value for simple games is provided by Dubey (1975). The essence of this characterization is the transfer property. A one-point solution concept $f: \mathrm{G}^{N} \rightarrow \mathbb{R}^{N}$ on a class $\mathrm{G}^{N} \subseteq \mathrm{TU}^{N}$ such that for all $v, w \in \mathrm{G}^{N}$ also $\max \{v, w\} \in \mathrm{G}^{N}$ and $\min \{v, w\} \in \mathrm{G}^{N}$, satisfies

- the transfer property if

$$
f(\max \{v, w\})+f(\min \{v, w\})=f(v)+f(w)
$$

for all $v, w \in \mathrm{G}^{N}{ }^{8}$

Dubey (1975) proved that the unique one-point solution concept on $\mathrm{SI}^{N}$ that satisfies efficiency, symmetry, the dummy property and the transfer property is the Shapley value (Shapley and Shubik 1954). The aim of this section is to see if the transfer property can also be used for a characterization for three-valued simple games.

First we formulate the properties above to fit on the class $\mathrm{TSI}^{N}$. A one-point solution concept $f: \operatorname{TSI}^{N} \rightarrow \mathbb{R}^{N}$ satisfies

- efficiency if $\sum_{i \in N} f_{i}(v)=2$ for all $v \in \mathrm{TSI}^{N}$.

- symmetry if $f_{i}(v)=f_{j}(v)$ for all $v \in \operatorname{TSI}^{N}$ and every pair $i, j \in N$ of symmetric players, where players $i, j \in N$ are symmetric in $v$ if

$$
v(S \cup\{i\})=v(S \cup\{j\}),
$$

for all $S \subseteq N \backslash\{i, j\}$.

- the dummy property if $f_{i}(v)=v(\{i\})$ for all $v \in \mathrm{TSI}^{N}$ and every dummy player $i \in N$ in $v$, where player $i \in N$ is a dummy player in $v$ if

$$
v(S \cup\{i\})=v(S)+v(\{i\}),
$$

for all $S \subseteq N \backslash\{i\}$.

The combination of the four properties efficiency, symmetry, dummy property and transfer property ${ }^{9}$ is not sufficient to characterize the Shapley value on the class of

\footnotetext{
8 The games $\max \{v, w\}$ and $\min \{v, w\}$ are defined by $(\max \{v, w\})(S)=\max \{v(S), w(S)\}$ and $(\min \{v, w\})(S)=\min \{v(S), w(S)\}$, for all $S \subseteq N$.

9 If $v, w \in \operatorname{TSI}^{N}$, then both $\max \{v, w\}$ and $\min \{v, w\}$ belong to $\operatorname{TSI}^{N}$ too.
} 
three-valued simple games: see Example A.1 in Appendix A. To obtain a characterization of $\Phi$ on $\mathrm{TSI}^{N}$, we introduce an additional fifth axiom: unanimity level efficiency.

A one-point solution concept $f: \mathrm{TSI}^{N} \rightarrow \mathbb{R}^{N}$ satisfies

- unanimity level efficiency if

$$
\sum_{i \in S} f_{i}\left(u_{S, T}\right)=1+\frac{1}{2} \sum_{i \in S} f_{i}\left(u_{T, T}\right),
$$

for all $S, T \in 2^{N} \backslash\{\emptyset\}$ with $S \subset T$.

Unanimity level efficiency intuitively states that in a double unanimity game $u_{S, T}$ with $S \subset T$ the players in $S$ can allocate a payoff of 1 between themselves, while for the remaining payoff of 1 (assuming efficiency) the players in $S$ and $T \backslash S$ are treated equally. Formally, the unanimity level efficiency axiom compares the aggregate payoff of coalition $S$ within a double unanimity game $u_{S, T}$, with $S \subset T$, to half of the payoffs to $S$ in the double unanimity game $u_{T, T}$. Note that the double unanimity game $u_{T, T}$ is a rescaling of the unanimity game $u_{T}$ and half of the payoffs to $S$ in the double unanimity game $u_{T, T}$ equals the payoffs to $S$ in the unanimity game $u_{T}$.

The combination of the axioms of efficiency, symmetry, the dummy property, the transfer property and unanimity level efficiency fully determines the Shapley value for a three-valued simple game.

Theorem 4.1 The Shapley value $\Phi$ is the unique one-point solution concept on TSI $^{N}$ satisfying the axioms efficiency, symmetry, the dummy property, the transfer property and unanimity level efficiency. ${ }^{10,11}$

Proof ${ }^{12}$ We first prove that, on $\mathrm{TSI}^{N}$, the Shapley value $\Phi$ satisfies the five axioms mentioned in the theorem. From Shapley (1953) it follows that $\Phi$ satisfies efficiency, symmetry and the dummy property on $\mathrm{TU}^{N}$. Moreover, from Dubey (1975) it follows that $\Phi$ satisfies the transfer property on $\mathrm{TU}^{N}$. Hence, $\Phi$ also satisfies efficiency, symmetry, the dummy property and the transfer property on $\operatorname{TSI}^{N}$, and thus it only remains to prove that $\Phi$ satisfies unanimity level efficiency on $\operatorname{TSI}^{N}$. Note that, for $S, T \in 2^{N}$ with $S \subset T$, we have

$$
\Phi_{i}\left(u_{S, T}\right)= \begin{cases}\frac{1}{|S|}+\frac{1}{|T|} & \text { if } i \in S \\ \frac{1}{|T|} & \text { if } i \in T \backslash S, \\ 0 & \text { otherwise, }\end{cases}
$$

and

$$
\Phi_{i}\left(u_{T, T}\right)= \begin{cases}\frac{2}{|T|} & \text { if } i \in T \\ 0 & \text { otherwise }\end{cases}
$$

\footnotetext{
10 A proof of the logical independence of the five axioms for $|N|=2$ can be found in Appendix A.

11 This characterization can be generalized for three-valued TU-games with coalitional values 0,1 or $\beta$ with $\beta>1$.

12 The proof is similar to the proof of Theorem II in Dubey (1975).
} 
for all $i \in N$. Consequently,

$$
\sum_{i \in S} f_{i}\left(u_{S, T}\right)-\frac{1}{2} \sum_{i \in S} f_{i}\left(u_{T, T}\right)=|S|\left(\frac{1}{|S|}+\frac{1}{|T|}\right)-\frac{1}{2}|S|\left(\frac{2}{|T|}\right)=1 .
$$

To finish the proof, we show that the five axioms exactly fix the allocation vector prescribed by the solution concept for every three-valued simple game. Let $f: \operatorname{TSI}^{N} \rightarrow \mathbb{R}^{N}$ be a one-point solution concept that satisfies efficiency, symmetry, the dummy property, the transfer property and unanimity level efficiency on $\operatorname{TSI}^{N}$. Let $v \in \operatorname{TSI}^{N}$ and define

$$
\begin{aligned}
& \operatorname{MWC}_{1}(v)=\{S \subset N \mid v(S)=1 \text { and } T \subset S \Rightarrow v(T)=0\}, \\
& \operatorname{MWC}_{2}(v)=\{S \subset N \mid v(S)=2 \text { and } T \subset S \Rightarrow v(T) \in\{0,1\}\},
\end{aligned}
$$

and set

$$
\operatorname{MWC}(v)=\operatorname{MWC}_{1}(v) \cup \operatorname{MWC}_{2}(v) .
$$

Observe the following:

(i) If $|\mathrm{MWC}(v)|=0$, then $v=u_{N, N}$. From efficiency and symmetry it follows that

$$
f\left(u_{N, N}\right)=\frac{2}{|N|} e^{N},
$$

and thus $f\left(u_{N, N}\right)$ is uniquely determined.

(ii) If $|\mathrm{MWC}(v)|=1$, then we have either $\left|\mathrm{MWC}_{1}(v)\right|=0$ and $\left|\mathrm{MWC}_{2}(v)\right|=1$, or $\left|\mathrm{MWC}_{1}(v)\right|=1$ and $\left|\mathrm{MWC}_{2}(v)\right|=0$.

If $\left|\mathrm{MWC}_{1}(v)\right|=0$ and $\left|\mathrm{MWC}_{2}(v)\right|=1$, then $v=u_{T, T}$ for some $T \subset N$. From efficiency, symmetry, and the dummy property it follows that $f\left(u_{T, T}\right)=\frac{2}{|T|} e^{T}$ and thus $f\left(u_{T, T}\right)$ is uniquely determined.

On the other hand, if $\left|\mathrm{MWC}_{1}(v)\right|=1$ and $\left|\mathrm{MWC}_{2}(v)\right|=0$, then $v=u_{S, N}$ for some $S \subset N$. From efficiency and symmetry together with unanimity level efficiency it follows that

$$
\sum_{i \in S} f\left(u_{S, N}\right)=1+\frac{1}{2} \sum_{i \in S} f_{i}\left(u_{N, N}\right)=1+\frac{|S|}{|N|}
$$

Then, due to efficiency and symmetry, we know $f\left(u_{S, N}\right)=\frac{1}{|S|} e^{S}+\frac{1}{|N|} e^{N}$ and thus $f\left(u_{S, N}\right)$ is uniquely determined.

Now let $v \in \operatorname{TSI}^{N}$ such that $|\operatorname{MWC}(v)|=m$ with $m \geq 2$. We use induction to show that $f(v)$ is uniquely determined. Assume that $f(w)$ is uniquely determined for all $w \in \operatorname{TSI}^{N}$ with $|\operatorname{MWC}(w)|<m$. Set $\operatorname{MWC}_{1}(v)=\left\{S_{1}, S_{2}, \ldots, S_{p}\right\}$ and 
$\operatorname{MWC}_{2}(v)=\left\{T_{1}, T_{2}, \ldots, T_{q}\right\}$ with $p+q=m$. Note that from monotonicity of $v$ it follows that

$$
T_{j} \nsubseteq S_{i}
$$

for all $i \in\{1, \ldots, p\}$ and $j \in\{1, \ldots, q\}$. We distinguish between two cases: $q=0$ and $q>0$.

Case 1: $q=0$, i.e., $\left|\mathrm{MWC}_{2}(v)\right|=0$ and $p=m$. Define

$$
w=\max \left\{u_{S_{2}, N}, \ldots, u_{S_{p}, N}\right\} \quad \text { and } \quad w^{\prime}=\min \left\{u_{S_{1}, N}, w\right\} .
$$

Then, $w, w^{\prime} \in \operatorname{TSI}^{N}$ and

$$
w(S)= \begin{cases}2 & \text { if } S=N, \\ 1 & \text { if there exists an } i \in\{2, \ldots, p\} \quad \text { such that } S_{i} \subseteq S, \\ 0 & \text { otherwise, }\end{cases}
$$

while

$$
w^{\prime}(S)= \begin{cases}2 & \text { if } S=N, \\ 1 & \text { if there exists an } i \in\{2, \ldots, p\} \quad \text { such that } S_{1} \cup S_{i} \subseteq S, \\ 0 & \text { otherwise. }\end{cases}
$$

Consequently, we have $\operatorname{MWC}_{1}(w)=\left\{S_{2}, S_{3}, \ldots, S_{p}\right\}$ and $\operatorname{MWC}_{2}(w)=\emptyset$, and thus

$$
|\mathrm{MWC}(w)|=p-1=m-1
$$

Moreover, $\operatorname{MWC}_{1}\left(w^{\prime}\right) \subseteq\left\{S_{1} \cup S_{2}, S_{1} \cup S_{3}, \ldots, S_{1} \cup S_{p}\right\}$ and $\operatorname{MWC}_{2}\left(w^{\prime}\right)=\emptyset$, and thus

$$
\left|\operatorname{MWC}\left(w^{\prime}\right)\right| \leq p-1=m-1
$$

Since $v=\max \left\{u_{S_{1}, N}, \ldots, u_{S_{p}, N}\right\}=\max \left\{u_{S_{1}, N}, w\right\}$ and by the transfer property, we have

$$
f\left(u_{S_{1}, N}\right)+f(w)=f\left(\max \left\{u_{S_{1}, N}, w\right\}\right)+f\left(\min \left\{u_{S_{1}, N}, w\right\}\right)=f(v)+f\left(w^{\prime}\right),
$$

and thus

$$
f(v)=f(w)+f\left(u_{S_{1}, N}\right)-f\left(w^{\prime}\right)
$$

Since by our induction hypothesis the right-hand side is uniquely determined, $f(v)$ is uniquely determined. 
Case 2: $q>0$, i.e., $\left|\mathrm{MWC}_{2}(v)\right|>0$. Define

$$
w=\max \left\{u_{S_{1}, N}, \ldots, u_{S_{p}, N}, u_{T_{2}, T_{2}}, \ldots, u_{T_{q}, T_{q}}\right\} \quad \text { and } \quad w^{\prime}=\min \left\{u_{T_{1}, T_{1}}, w\right\}
$$

Then, $w, w^{\prime} \in \operatorname{TSI}^{N}$ and

$$
w(S)= \begin{cases}2 & \text { if there exists an } i \in\{2, \ldots, q\} \quad \text { such that } T_{i} \subseteq S, \\ 1 & \text { if there exists an } i \in\{1, \ldots, p\} \quad \text { such that } S_{i} \subseteq S \\ & \text { and } T_{j} \nsubseteq S \text { for all } j \in\{2, \ldots, q\} \\ 0 & \text { otherwise, }\end{cases}
$$

while

$$
w^{\prime}(S)= \begin{cases}2 & \text { if there exists an } i \in\{2, \ldots, q\} \quad \text { such that } T_{1} \cup T_{i} \subseteq S, \\ 1 & \text { if there exists an } i \in\{1, \ldots, p\} \quad \text { such that } T_{1} \cup S_{i} \subseteq S \\ \text { and } T_{j} \nsubseteq S \text { for all } j \in\{2, \ldots, q\} & \\ 0 & \text { otherwise. }\end{cases}
$$

Since $T_{j} \nsubseteq S_{i}$ for all $i \in\{1, \ldots, p\}$ and $j \in\{1, \ldots, q\}$, we have $\operatorname{MWC}_{1}(w)=$ $\left\{S_{1}, S_{2}, \ldots, S_{p}\right\}$ and $\mathrm{MWC}_{2}(w)=\left\{T_{2}, T_{3}, \ldots, T_{q}\right\}$, and thus

$$
|\mathrm{MWC}(w)|=p+(q-1)=m-1 .
$$

Moreover, $\operatorname{MWC}_{1}\left(w^{\prime}\right) \subseteq\left\{T_{1} \cup S_{1}, T_{1} \cup S_{2}, \ldots, T_{1} \cup S_{p}\right\}$ and $\operatorname{MWC}_{2}\left(w^{\prime}\right) \subseteq\left\{T_{1} \cup\right.$ $T_{2}, T_{1} \cup T_{3}, \ldots, T_{1} \cup T_{q}$ \}, and thus

$$
\left|\mathrm{MWC}\left(w^{\prime}\right)\right| \leq p+(q-1)=m-1
$$

Since $v=\max \left\{u_{S_{1}, N}, \ldots, u_{S_{p}, N}, u_{T_{1}, T_{1}}, u_{T_{2}, T_{2}}, \ldots, u_{T_{q}, T_{q}}\right\}=\max \left\{u_{T_{1}, T_{1}}, w\right\}$ and by the transfer property, we have

$$
f\left(u_{T_{1}, T_{1}}\right)+f(w)=f\left(\max \left\{u_{T_{1}, T_{1}}, w\right\}\right)+f\left(\min \left\{u_{T_{1}, T_{1}}, w\right\}\right)=f(v)+f\left(w^{\prime}\right),
$$

and thus

$$
f(v)=f(w)+f\left(u_{T_{1}, T_{1}}\right)-f\left(w^{\prime}\right)
$$

Since by our induction hypothesis the right-hand side is uniquely determined, $f(v)$ is uniquely determined too.

Applications of the Shapley value for measuring the power in legislative procedures are abundant in the literature. For instance, Bilbao et al. (2002) considered the legislative procedure of the EU-27 Council by means of a combinatorial method based on generating functions for computing the Shapley value efficiently. Likewise, Hausken and Mohr (2001) considered the legislative procedure in the European Council of 
Ministers. In particular, they decomposed the Shapley value into a matrix for which the elements in each row and in each column of the matrix sum up to the Shapley value of the corresponding player.

Three-valued simple games can be used to more adequately model a bicameral legislature, in which the legislators are divided into two separate houses, the lower house and the upper house, and a bill has to be approved by both houses. Example 4.1 shows how a three-valued simple game can be used to model the bicameral legislature in the Netherlands.

Example 4.1 In the bicameral legislature of the Netherlands, the States General of the Netherlands, the lower house is called the House of Representatives and the upper house is called the Senate. The House of Representatives consists of 150 members and the Senate consists of 75 members. The members of the Senate and House of Representatives each represent a political party. The members of the House of Representatives are elected directly by the Dutch citizens. The members of the Senate, however, are elected indirectly by the members of the provincial councils, who, in turn, are elected directly by the Dutch citizens.

The House of Representatives can accept or reject a bill. A bill is accepted by the House of Representatives only if there is a majority in favor. When a bill is accepted by the House of Representatives it is forwarded to the Senate. Subsequently the Senate can also accept or reject a bill, again via majority voting. If the bill is also accepted by the Senate, then the bill becomes a law. ${ }^{13}$ Note that the House of Representatives and the Senate are not symmetric in the sense that the House of Representatives also has the right to propose or to revise a bill, while the Senate does not have this right.

The party breakdown of the House of Representatives and the Senate in September 2016 can be found in Table 2, where $a_{i}$ and $b_{i}$ denote the number of members in the House of Representatives and Senate, respectively, for party $i$.

The legislature of the Netherlands can be modelled by means of the following simple game $w \in \mathrm{SI}^{N}$ where the set of players $N$ is the set of parties and the value $w(S)$ of a coalition $S \subseteq N$ equals:

- 1 , if the members of all parties in the coalition form a majority in both the House of Representatives and Senate,

- 0 , otherwise.

Hence, we have

$$
w(S)= \begin{cases}1 & \text { if } \sum_{i \in S} a_{i} \geq 76 \quad \text { and } \quad \sum_{i \in S} b_{i} \geq 38 \\ 0 & \text { otherwise }\end{cases}
$$

for all $S \subseteq N$. Note, however, that this game does not take into account the asymmetry of the House of Representatives and the Senate.

\footnotetext{
13 Note that in the current (2016) composition of the States General of the Netherlands, the government (kabinet-Rutte II, a coalition of VVD and PvdA) forms a majority in the House of Representatives, but not in the Senate. Hence, in order to accept a bill, the government needs the support of other political parties as well.
} 
Table 2 Party breakdown of the House of Representatives and the Senate of the Netherlands (updated to September 2016), sources: https://www.house ofrepresentatives.nl/member s_of_parliament/parliamenta ry_parties and https://www. eerstekamer.nl/begrip/english_2

\begin{tabular}{lrr}
\hline Party & $a_{i}$ & $b_{i}$ \\
\hline VVD & 40 & 13 \\
PvdA & 36 & 8 \\
SP & 15 & 9 \\
CDA & 13 & 12 \\
D66 & 12 & 10 \\
PVV & 12 & 9 \\
CU & 5 & 3 \\
GL & 4 & 4 \\
SGP & 3 & 2 \\
PvdD & 2 & 2 \\
GrKO & 2 & 0 \\
GrBvK & 2 & 0 \\
50PLUS & 1 & 2 \\
Houwers & 1 & 0 \\
Klein & 1 & 0 \\
Van Vliet & 1 & 0 \\
OSF & 0 & 1 \\
Total & 150 & 75 \\
\hline & &
\end{tabular}

Alternatively, a three-valued simple game does offer the opportunity to model the asymmetric bicameral legislature of the Netherlands into a single TU-game. Consider the three-valued simple game $v \in \mathrm{TSI}^{N}$ where the set of players $N$ is again the set of parties. Now, the value $v(S)$ of a coalition $S \subseteq N$ equals:

- 2 , if the members of all parties in the coalition form a majority in both the House of Representatives and Senate,

- 1 , if the members of all parties in the coalition form a majority in the House of Representatives but not in the Senate,

- 0 , otherwise.

Hence, we have

$$
v(S)= \begin{cases}2 \quad \text { if } \quad \sum_{i \in S} a_{i} \geq 76 \quad \text { and } \quad & \sum_{i \in S} b_{i} \geq 38 \\ 1 \quad & \text { if } \sum_{i \in S} a_{i} \geq 76 \quad \text { and } \quad \sum_{i \in S} b_{i}<38 \\ 0 & \text { otherwise }\end{cases}
$$

for all $S \subseteq N$. For example, since $a_{\mathrm{VVD}}+a_{\mathrm{PvdA}}=76 \geq 76$ and $b_{\mathrm{VVD}}+b_{\mathrm{PvdA}}=$ $21<38$, we have $v(\{$ VVD, PvdA $\})=1$. Hence, the parties VVD and PvdA have a majority in the House of Representatives, but not in the Senate.

Table 3 provides the rankings according to the Shapley values of the resulting simple and three-valued simple game. Note that generally speaking there are no major 
Table 3 The rankings of the parties in the bicameral legislature of the Netherlands based on $\Phi(w)$ and $\Phi(v)$, respectively

\begin{tabular}{|c|c|c|c|}
\hline Ranking & Party & Ranking & Party \\
\hline \multicolumn{2}{|c|}{ (a) Ranking according to $\Phi(w)$} & \multicolumn{2}{|c|}{ (b) Ranking according to $\Phi(v)$} \\
\hline 1. & VVD & 1. & VVD \\
\hline 2. & PvdA & 2. & PvdA \\
\hline 3. & CDA & 3. & SP \\
\hline 4. & SP & 4. & CDA \\
\hline 5. & D66 & 5. & D66 \\
\hline 6. & PVV & 6. & PVV \\
\hline 7. & GL & 7. & $\mathrm{CU}$ \\
\hline 8. & $\mathrm{CU}$ & 8. & GL \\
\hline 9. & SGP & 9. & SGP \\
\hline 10. & PvdD & 10. & PvdD \\
\hline 11. & 50PLUS & 11. & 50PLUS \\
\hline 12. & GrKO & 12. & GrKO \\
\hline 12. & GrBvK & 12. & GrBvK \\
\hline 14. & OSF & 14. & Houwers \\
\hline 15. & Houwers & 14. & Klein \\
\hline 15. & Klein & 14. & Van Vliet \\
\hline 15. & Van Vliet & 16. & OSF \\
\hline
\end{tabular}

differences in the resulting rankings. So, in this case, the basic modelling via a simple game can be seen as a good approximation.

Open Access This article is distributed under the terms of the Creative Commons Attribution 4.0 International License (http://creativecommons.org/licenses/by/4.0/), which permits unrestricted use, distribution, and reproduction in any medium, provided you give appropriate credit to the original author(s) and the source, provide a link to the Creative Commons license, and indicate if changes were made.

\section{Appendix}

In order to prove the logical independence of the five axioms efficiency, symmetry, the dummy property, the transfer property and unanimity level efficiency on $\mathrm{TSI}^{N}$ with $|N|=2$, we provide five examples to show the necessity of each of the five properties. Note that if $N=\{1,2\}$, then there are exactly nine different three-valued simple games. These games are listed in Table 4.

Since players 1 and 2 are symmetric players in $v_{1}, v_{5}, v_{9}$ and there are no symmetric players in the other games, the symmetry property of a solution $f$ is satisfied if and only if

$$
f_{1}\left(v_{1}\right)=f_{2}\left(v_{1}\right)
$$




$$
\begin{aligned}
& f_{1}\left(v_{5}\right)=f_{2}\left(v_{5}\right), \\
& f_{1}\left(v_{9}\right)=f_{2}\left(v_{9}\right) .
\end{aligned}
$$

Likewise, since player 1 is a dummy player in $v_{3}$ and player 2 is a dummy player in $v_{7}$ and there are no dummy players in the other games, the dummy property of a solution $f$ is satisfied if and only if

$$
\begin{aligned}
& f_{1}\left(v_{3}\right)=0, \\
& f_{2}\left(v_{7}\right)=0 .
\end{aligned}
$$

Moreover, the transfer property boils down to the following nine equalities:

$$
\begin{aligned}
& f\left(v_{2}\right)+f\left(v_{4}\right)=f\left(v_{1}\right)+f\left(v_{5}\right), \\
& f\left(v_{2}\right)+f\left(v_{7}\right)=f\left(v_{1}\right)+f\left(v_{8}\right), \\
& f\left(v_{3}\right)+f\left(v_{4}\right)=f\left(v_{1}\right)+f\left(v_{6}\right), \\
& f\left(v_{3}\right)+f\left(v_{5}\right)=f\left(v_{2}\right)+f\left(v_{6}\right), \\
& f\left(v_{3}\right)+f\left(v_{7}\right)=f\left(v_{1}\right)+f\left(v_{9}\right), \\
& f\left(v_{3}\right)+f\left(v_{8}\right)=f\left(v_{2}\right)+f\left(v_{9}\right), \\
& f\left(v_{5}\right)+f\left(v_{7}\right)=f\left(v_{4}\right)+f\left(v_{8}\right), \\
& f\left(v_{6}\right)+f\left(v_{7}\right)=f\left(v_{4}\right)+f\left(v_{9}\right), \\
& f\left(v_{6}\right)+f\left(v_{8}\right)=f\left(v_{5}\right)+f\left(v_{9}\right) .
\end{aligned}
$$

Finally, the unanimity level efficiency property boils down to the following two equalities:

$$
\begin{aligned}
& f_{2}\left(v_{2}\right)=1+\frac{1}{2} f_{2}\left(v_{1}\right), \\
& f_{1}\left(v_{4}\right)=1+\frac{1}{2} f_{1}\left(v_{1}\right) .
\end{aligned}
$$

Table 4 All possible two-person three-valued simple games

\begin{tabular}{llll}
\hline$S$ & $\{1\}$ & $\{2\}$ & $\{1,2\}$ \\
\hline$v_{1}(S)$ & 0 & 0 & 2 \\
$v_{2}(S)$ & 0 & 1 & 2 \\
$v_{3}(S)$ & 0 & 2 & 2 \\
$v_{4}(S)$ & 1 & 0 & 2 \\
$v_{5}(S)$ & 1 & 1 & 2 \\
$v_{6}(S)$ & 1 & 2 & 2 \\
$v_{7}(S)$ & 2 & 0 & 2 \\
$v_{8}(S)$ & 2 & 1 & 2 \\
$v_{9}(S)$ & 2 & 2 & 2 \\
\hline
\end{tabular}


Example A.1 (Necessity of unanimity level efficiency) Consider the one-point solution concept $f$, where

$$
\begin{aligned}
& f\left(v_{1}\right)=f\left(v_{2}\right)=f\left(v_{4}\right)=f\left(v_{5}\right)=f\left(v_{9}\right)=(1,1), \\
& f\left(v_{3}\right)=f\left(v_{6}\right)=(0,2), \\
& f\left(v_{7}\right)=f\left(v_{8}\right)=(2,0) .
\end{aligned}
$$

Clearly $f\left(v_{6}\right) \neq \Phi\left(v_{6}\right)$ while $f$ satisfies efficiency, symmetry, the dummy property and the transfer property, but does not satisfy unanimity level efficiency.

Example A.2 (Necessity of the transfer property) Consider the one-point solution concept $f$, where

$$
\begin{aligned}
& f\left(v_{1}\right)=f\left(v_{5}\right)=f\left(v_{9}\right)=(1,1), \\
& f\left(v_{2}\right)=\left(\frac{1}{2}, 1 \frac{1}{2}\right), \\
& f\left(v_{3}\right)=f\left(v_{6}\right)=(0,2), \\
& f\left(v_{4}\right)=\left(1 \frac{1}{2}, \frac{1}{2}\right), \\
& f\left(v_{7}\right)=f\left(v_{8}\right)=(2,0) .
\end{aligned}
$$

Clearly $f\left(v_{6}\right) \neq \Phi\left(v_{6}\right)$ while $f$ satisfies efficiency, symmetry, the dummy property and unanimity level efficiency, but does not satisfy the transfer property.

Example A.3 (Necessity of the dummy property) Consider the one-point solution concept $f$, where

$$
\begin{aligned}
& f\left(v_{1}\right)=f\left(v_{5}\right)=f\left(v_{6}\right)=f\left(v_{8}\right)=f\left(v_{9}\right)=(1,1), \\
& f\left(v_{2}\right)=f\left(v_{3}\right)=\left(\frac{1}{2}, 1 \frac{1}{2}\right), \\
& f\left(v_{4}\right)=f\left(v_{7}\right)=\left(1 \frac{1}{2}, \frac{1}{2}\right) .
\end{aligned}
$$

Clearly $f\left(v_{6}\right) \neq \Phi\left(v_{6}\right)$ while $f$ satisfies efficiency, symmetry, the transfer property and unanimity level efficiency, but does not satisfy the dummy property.

Example A.4 (Necessity of symmetry) Consider the one-point solution concept $f$, where

$$
\begin{aligned}
& f\left(v_{1}\right)=f\left(v_{4}\right)=f\left(v_{7}\right)=(2,0), \\
& f\left(v_{2}\right)=f\left(v_{5}\right)=f\left(v_{8}\right)=(1,1), \\
& f\left(v_{3}\right)=f\left(v_{6}\right)=f\left(v_{9}\right)=(0,2),
\end{aligned}
$$

Clearly $f\left(v_{6}\right) \neq \Phi\left(v_{6}\right)$ while $f$ satisfies efficiency, the dummy property, the transfer property and unanimity level efficiency, but does not satisfy symmetry. 
Example A.5 (Necessity of efficiency) Consider the one-point solution concept $f$, where

$$
\begin{aligned}
& f\left(v_{1}\right)=f\left(v_{2}\right)=f\left(v_{4}\right)=f\left(v_{5}\right)=f\left(v_{9}\right)=(2,2), \\
& f\left(v_{3}\right)=f\left(v_{6}\right)=(0,4), \\
& f\left(v_{7}\right)=f\left(v_{8}\right)=(4,0),
\end{aligned}
$$

Clearly $f\left(v_{6}\right) \neq \Phi\left(v_{6}\right)$ while $f$ satisfies symmetry, the dummy property, the transfer property and unanimity level efficiency, but does not satisfy efficiency.

\section{References}

Bilbao, J., Fernández, J., Jiménez, N., \& López, J. (2002). Voting power in the European Union enlargement. European Journal of Operational Research, 143, 181-196.

Deng, X., Ibaraki, T., \& Nagamochi, H. (1999). Algorithmic aspects of the core of combinatorial optimization games. Mathematics of Operations Research, 24, 751-766.

Dubey, P. (1975). On the uniqueness of the Shapley value. International Journal of Game Theory, 4, 131139.

Felsenthal, D., \& Machover, M. (1997). Ternary voting games. International Journal of Game Theory, 26, $335-351$.

Freixas, J. (2005a). Banzhaf measures for games with several levels of approval in the input and output. Annals of Operations Research, 137, 45-66.

Freixas, J. (2005b). The Shapley-Shubik power index for games with several levels of approval in the input and output. Decision Support Systems, 39, 185-195.

Freixas, J. (2012). Probabilistic power indices for voting rules with abstention. Mathematical Social Sciences, 64, 89-99.

Freixas, J., \& Zwicker, W. (2003). Weighted voting, abstention, and multiple levels of approval. Social Choice and Welfare, 21, 399-431.

Gibbard, A. (1973). Manipulation of voting schemes: A general result. Econometrica: Journal of the Econometric Society, 41, 587-601.

Hausken, K., \& Mohr, M. (2001). The value of a player in $n$-person games. Social Choice and Welfare, 18, 465-483.

Hsiao, C., \& Raghavan, T. (1993). Shapley value for multichoice cooperative games, Games and Economic Behavior, 5, 240-256.

Ichiishi, T. (1981). Super-modularity: Applications to convex games and the greedy algorithm for LP. Journal of Economic Theory, 25, 283-286.

Musegaas, M., Borm, P., \& Quant, M. (2016). Simple and three-valued simple minimum coloring games. Mathematical Methods of Operations Research, 84, 239-258.

Shapley, L. (1953). A value for n-person games. Annals of Mathematics Studies, 28, 307-317.

Shapley, L. (1971). Cores of convex games. International Journal of Game Theory, 1, 11-26.

Shapley, L., \& Shubik, M. (1954). A method for evaluating the distribution of power in a committee system. American Political Science Review, 48, 787-792.

von Neumann, J., \& Morgenstern, O. (1944). Theory of Games and Economic Behavior. Princeton: Princeton University Press. 